\title{
Some oddities of light-cone dynamics
}

Orit Levin and Asher Peres

Department of Physics, Technion-Israel Institute of Technology, 32000 Haifa, Israel

Received December 1993

Abstract. A classical canonical transformation can convert the Poincaré group generators from the usual "instant form" into expressions associated with a lightcone. However, there is no consistent Hamiltonian dynamics for quantum wave functions on a light-cone, because the radial momentum operator is not self-adjoint.

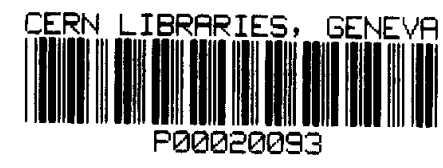

Classification: 03.65 
In a classic article, Dirac (1949) investigated the conditions for compatibility of the canonical equations of motion with the requirements of special relativity. In essence, Dirac's argument was that if a canonical formulation is possible in one Lorentz frame, it should be possible in every Lorentz frame (by the principle of relativity). Therefore a Lorentz transformation must be a canonical transformation of the dynamical variables. In particular, an infinitesimal Lorentz transformation has ten generators, namely the Hamiltonian $H$ (translation in time), the total momentum $\mathbf{P}$ (translation in space), the total angular momentum $\mathbf{J}$ (rotations), and the boost $\mathbf{K}$ (pure Lorentz transformations). Their Poisson brackets can be derived from the way the Poincaré group acts on spacetime coordinates.

An explicit form of these generators, for a single free particle of mass $m$, is Dirac's "instant form," namely (at time $t=0$, and with natural units, $c=1$ )

$$
\begin{aligned}
& H=\left(\mathbf{p}^{2}+m^{2}\right)^{1 / 2}, \\
& \mathbf{P}=\mathbf{p}, \\
& \mathbf{J}=\mathbf{q} \times \mathbf{p}, \\
& \mathbf{K}=-\mathbf{q} H,
\end{aligned}
$$

where $\mathbf{q}$ denotes the three Cartesian coordinates, and the components of $\mathbf{p}$ are the conjugate momenta. The canonical variables $\mathbf{q}$ thus have the physical meaning of geometric coordinates in Euclidean space. The generators $\mathbf{P}$ and $\mathbf{J}$ also have a purely geometric meaning: they translate and rotate a constant- $t$ hyperplane. On the other hand, $H$ and $\mathbf{K}$ explicitly involve the mass $m$ and have a dynamical meaning: they transform dynamical variables from a constant- $t$ hyperplane to a neighboring parallel hyperplane, or to a slightly tilted hyperplane, respectively.

Dirac (1949) also pointed out that there were other possible forms for these ten generators. In particular, $\mathbf{J}$ and $\mathbf{K}$ could be purely geometric (independent of the mass), while $H$ and $\mathbf{P}$ would involve $m$ and have a dynamical meaning. With this "point form" representation, the physical meaning of the canonical coordinates is that of positions on a Lorentz invariant hyperboloid or, as a limiting case, on a light-cone. A third form of relativistic canonical dynamics, associated with a light front (such as $x+t=$ const.) was also discussed by Dirac.

The explicit form of the generators for a light-cone is 


$$
\begin{aligned}
& H^{\prime}=\left(k^{2}+m^{2}\right) / 2 k_{r}, \\
& \mathbf{P}^{\prime}=\mathbf{k}-H^{\prime} \mathbf{r} / r, \\
& \mathbf{J}^{\prime}=\mathbf{r} \times \mathbf{k}, \\
& \mathbf{K}^{\prime}=-r \mathbf{k},
\end{aligned}
$$

where $\mathbf{r}$ and $\mathbf{k}$ denote a new set of canonical coordinates and momenta, and where $k_{r}=(\mathbf{k} \cdot \mathbf{r}) / r$ is constrained to be non-negative (this restriction of phase space will be discussed below). These relations are valid for a past light-cone. For a future light-cone, $k_{r} \leq 0$. We then have $H^{\prime}=-\left(k^{2}+m^{2}\right) / 2 k_{r}$ and the minus signs in Eqs. (6) and (8) are replaced by + signs.

A canonical transformation which converts the Poincaré group generators from the instant form (1-4) to the light-cone form (5-8) was found by Derrick (1987):

$$
\begin{aligned}
& \mathbf{q}=\frac{2(\mathbf{k} \cdot \mathbf{r})}{k^{2}+m^{2}} \mathbf{k}, \\
& \mathbf{p}=\mathbf{k}-\frac{k^{2}+m^{2}}{2(\mathbf{k} \cdot \mathbf{r})} \mathbf{r},
\end{aligned}
$$

which is also valid for the future light-cone. To prove that is canonical, Poisson brackets of the various $\mathbf{q}$ and $\mathbf{p}$ can be computed in terms of the $\mathbf{r}$ and $\mathbf{k}$ variables, and seen to have the correct values. It is easily verified that the substitutions (9) and (10) give $H=H^{\prime}, \mathbf{P}=\mathbf{P}^{\prime}$, etc. There is therefore a complete equivalence between the two forms of canonical dynamics.

The physical interpretation of this result is quite simple: if a free particle has position $\mathbf{q}$ and momentum $\mathbf{p}$ at time $t=0$, its world line crosses the past light cone $|\mathbf{q}|=-t$ at the point

$$
\mathbf{q}_{t}=\mathbf{q}+\mathbf{p} t / H=\mathbf{r}
$$

at a time given by

$$
t / H=-2(\mathbf{k} \cdot \mathbf{r}) /\left(k^{2}+m^{2}\right) .
$$

This may be seen by substitution of (9) and (10) into (11). It then follows from (5) that $\left|\mathbf{q}_{t}\right|=-t$, so that this point indeed lies on the past light-cone.

We must now examine the consistency of the constraint $(\mathbf{k} \cdot \mathbf{r}) \geq 0$. For this, we need to express $\mathbf{r}$ and $\mathbf{k}$ in terms of $\mathbf{q}$ and $\mathbf{p}$. From (9) and (10) we have 


$$
(\mathbf{p} \cdot \mathbf{q})\left(k^{2}+m^{2}\right)=(\mathbf{k} \cdot \mathbf{r})\left(k^{2}-m^{2}\right)
$$

and

$$
q^{2}\left(k^{2}-m^{2}\right)^{2}-4(\mathbf{p} \cdot \mathbf{q})^{2}\left(k^{2}-m^{2}\right)-4(\mathbf{p} \cdot \mathbf{q})^{2} m^{2}=0 .
$$

If $(\mathbf{p} \cdot \mathbf{q}) \neq 0$, the last equation has two roots with opposite signs:

$$
k^{2}-m^{2}=2 q^{-2}\left[(\mathbf{p} \cdot \mathbf{q})^{2} \pm(\mathbf{p} \cdot \mathbf{q}) \sqrt{(\mathbf{p} \cdot \mathbf{q})^{2}+q^{2} m^{2}}\right] .
$$

By choosing the $+\operatorname{sign}$ in (15), we force $\left(k^{2}-m^{2}\right)$ to have the same sign as $(\mathbf{p} \cdot \mathbf{q})$, and it then follows from (13) that $(\mathbf{k} \cdot \mathbf{r}) \geq 0$, as desired. We can then obtain $\mathbf{k}$ and $\mathbf{r}$ as single-valued functions of $\mathbf{p}$ and $\mathbf{q}$.

There is, however, another possibility. We may introduce a "second sheet" of phase space, corresponding to the minus sign in Eq. (15). On that sheet, $(\mathbf{k} \cdot \mathbf{r})<0$, and therefore $H^{\prime}<0$. We can nevertheless retain $H=H^{\prime}$ by introducing explicitly a new canonical variable $\epsilon$, whose value is 1 on the first sheet, and -1 on the second sheet. We assume, instead of Eq. (1), that $H=\epsilon\left(p^{2}+m^{2}\right)^{1 / 2}$, and the second sheet can then be interpreted in terms of Dirac's "holes" (antiparticles).

This completely solves the classical problem. Unfortunately, when quantisation is contemplated, we run into serious difficulties which were largely ignored by former authors (Peres 1968, Derrick 1987, Mosley and Farina 1992). The point is that the radial momentum operator, $k_{r}$, which explicitly appears in $H^{\prime}$, and has to satisfy $\left[r, k_{r}\right]=i$ in order to obtain the correct commutation relations of the Poincare group, is not self-adjoint and has no self-adjoint extension, because the spectrum of $r$ is bounded below (von Neumann 1955, Zhu and Klauder 1993). This is a special case of a general theorem: if $A$ and $B$ are self-adjoint operators satisfying $[A, B]=i$, the unitary operator $e^{i \lambda B}$ shifts the value of $A$ by an amount $\lambda$, and at the same time leaves the spectrum of $A$ invariant (since this is a unitary transformation). Therefore that spectrum must extend from $-\infty$ to $\infty$.

Thus, since $r \geq 0$, the conjugate operator $k_{r}$ is not self-adjoint and has no self-adjoint extension. It has no spectral decomposition, and is not physically observable. The same is true for $H^{\prime}$ given by Eq. (5). For example, it is impossible to divide the Hilbert space into two orthogonal subspaces with positive and negative $H^{\prime}$ (like the two sheets of the classical phase space). This difficulty does not arise with the instant-form Hamiltonian $H$ in Eq. (1), even if the latter is written in 
polar coordinates, because $H$ involves ${p_{q}}^{2}=(\mathbf{p} \cdot \mathbf{q})^{2} / q^{2}$. In quantum theory, this becomes $p_{q}{ }^{*} p_{q}$, which is a self-adjoint operator, even though $p_{q}$ itself is not (Riesz and Sz.-Nagy 1955, p. 313).

Nonetheless, quantisation of $H^{\prime}$ is possible: instead of using the operator $k_{r}$, which is not self-adjoint, we may take the positive square root $\left(k_{r}{ }^{*} k_{r}\right)^{1 / 2}$, which is a uniquely defined self-adjoint operator (Riesz and Sz.-Nagy 1955, pp. 263265). However, that square root does not satisfy the commutation relation $\left[r,\left(k_{r}{ }^{*} k_{r}\right)^{1 / 2}\right]=i$ (no self-adjoint operator can satisfy it) and if it is used for defining $H^{\prime}$, the latter fails to have the desired commutation relations with $\mathbf{P}^{\prime}$ and $\mathbf{K}^{\prime}$. It thus appears that there is no consistent Hamiltonian dynamics for quantum wave functions on a light-cone.

Work by OL was supported by the Technion Graduate School. Work by AP was supported by the Gerard Swope Fund and by the Fund for Encouragement of Research.

\section{References}

Derrick G H 1987 J. Math. Phys. 2864 and 1327

Dirac P A M 1949 Rev. Mod. Phys. 21392

Mosley S N and Farina J E G 1992 J. Phys. A: Math. Gen. 254673 and 4687

Peres A 1968 J. Math. Phys. 9785

Riesz F and Sz.-Nagy B 1955 Functional Analysis, Ungar, New York

von Neumann J 1955 Mathematical Foundations of Quantum Mechanics, Princeton Univ. Press, Princeton, pp. 139 and 168

Zhu C and Klauder J R 1993 Found. Phys. 23 617; Am. J. Phys. 61605 